\title{
Initial Examination of AltiKa's Individual Echoes
}

\author{
Graham D. Quartly ${ }^{1} \&$ Marcello Passaro ${ }^{2,3}$ \\ 1 - Plymouth Marine Laboratory (e: gqu@pml.ac.uk), 2 - GSNOCS, University of Southampton, 3 - ESA/ESRIN
}

This is an Accepted Manuscript of an article published by Taylor \& Francis, to appear in Marine Geodesy SARAL/AltiKa special issue. doi: 10.1080/01490419.2014.984882

\begin{abstract}
The AltiKa altimeter records the reflection of $\mathrm{K}_{\mathrm{a}}$-band radar pulses from the Earth's surface, with the commonly used waveform product involving the summation of 96 returns to provide average echoes at $40 \mathrm{~Hz}$. Occasionally there are 1-second recordings of the complex individual echoes (IEs), which facilitate the evaluation of on-board processing and offers the potential for new processing strategies. Our investigation of these IEs over the ocean confirms the on-board operations, whilst noting that data quantization limits the accuracy in the thermal noise region. By constructing average waveforms from 32 IEs at a time, and applying an innovative subwaveform retracker, we demonstrate that accurate height and wave height information can be retrieved from very short sections of data. Early exploration of the complex echoes reveals structure in the phase information similar to that noted for Envisat's IEs.
\end{abstract}

Keywords: AltiKa altimeter, $\mathrm{K}_{\mathrm{a}}$-band, Waveforms, Individual echoes, Phase analysis, Retracking, Coherence

\section{Introduction}

A radar altimeter provides information on the Earth's surface by emitting a series of very short radio-wave pulses and recording their reflected signal. In conventional (low-rate mode) altimetry, the signal recorded is the time record of the returned power, which is termed a "waveform". Over a supposedly homogeneous surface such as the ocean the expected signal is the convolution of three terms - the shape of the emitted pulse (often referred to as the "point target response"), the distance of points on a plane from a location above it (the "flat surface response"), and the probability distribution function of the height of reflecting facets on the sea surface (which is principally governed by the distribution of waves). An analytical expression exists for this expected signal (see light grey curve in Fig. 1a), parameterizing the waveform in terms of amplitude, slope of leading edge and distance to midpoint of the leading edge (Brown 1977; Hayne 1980). These are respectively related to the wind speed, wave height and range (from which sea surface height is determined).

However, the echo for any individual pulse (e.g. thin black line in Fig. 1a) will deviate from this expected smooth curve, as the returned signal at each bin (range interval within the waveform) will be due to the summation of reflections from a myriad of facets at the appropriate range, with the phase of these micro-reflections being randomly distributed. Consequently, for a single pulse, the realized power at a certain bin will be from a negative exponential distribution (Fig. 1b), whose mean is predicted by the convolution discussed earlier. Provided the signals are correctly aligned, the summation of the power from multiple echoes provides a convergence towards the expected shape.

Most physical ocean retrackers (i.e. algorithms to derive geophysical information from the waveforms) are based on the Brown-Hayne formulation (Brown 1977; Hayne 1980), with the slope of the leading edge being used to infer the significant wave height $\left(\mathrm{H}_{\mathrm{s}}\right)$, the position of its mid-power point corresponding to range, from which sea surface height is derived, and the amplitude contributing to the calculation of normalized backscatter, $\sigma^{0}$, from which wind speed is inferred. The slope of the trailing edge can be related to mispointing of the altimeter, though attempts to fit this parameter also affect the estimation of $\sigma^{0}$ (Quartly 2009).

AltiKa, launched in February 2013, is the first $\mathrm{K}_{\mathrm{a}}$-band altimeter, operating at $35.75 \mathrm{GHz}$, with a pulse repetition frequency of $\sim 3700 \mathrm{~Hz}$. As well as providing mean waveforms at $40 \mathrm{~Hz}$ (i.e. every 174 $\mathrm{m}$ along track), it has a mode that allows the collection and download of all the individual echoes (IE) within a 1 -second burst. This follows on from the use of such a mode at $\mathrm{K}_{\mathrm{u}}$-band in the operation of 
Envisat's altimeter. In this paper we take a preliminary look at some of these bursts of IEs from AltiKa. Section 2 describes the on-board processing, and Section 3 investigates its implementation to confirm that the design choices on extent and resolution of telemetered data have not adversely affected performance. Section 4 explores the accuracy of retracking at a finer spatial resolution, which could be of benefit over rapidly varying terrain e.g. leads in sea-ice or rivers less than $1 \mathrm{~km}$ in width. Section 5 looks at the phase information provided within IEs, which may suggest further approaches to improve retracking.

\section{AltiKa data collection and on-board processing}

Although conceptually altimeters emit very short radio pulses, in practice this is not possible because of the high peak power required in order to get a reasonable echo. Instead, as detailed in Chelton et al. (1989), they produce a chirp of the required bandwidth (500 MHz in the case of AltiKa), and at the time of the anticipated return signal they mix the receiver channel with an appropriately delayed facsimile of the original chirp. This copy is actually at a frequency offset from the original transmitted signal, so the combining of these two leads to a signal at the significantly lower beat frequency. Finally, a filter centred on the expected intermediate frequency is applied. This process of mixing with the expected signal, known as "deramping", thus first produces a signal in frequency space. This is registered as a series of 128 in-phase and quadrature (I and Q) components. A Fourier transform, plus power summation over returns, is required to produce the familiar delayed time echo shown in waveforms.

AltiKa emits $\sim 3700$ chirps per second, with the returns normally being summed in groups of 96 to produce average echoes (AEs) at the rate of $40 \mathrm{~Hz}$. As the altimeter recedes from or approaches the Earth, there will be marked changes in the travel time for the echo, with uncertainties in its prediction leading to varying positions of the pulses within the waveform window. To achieve a sharp pulse shape it is important that the leading edge of the individual echoes (IEs) have been lined up prior to summation. This is achieved in frequency space by multiplying each individual component by $\exp (-2 \pi \mathrm{j} \xi \mathrm{i} / \mathrm{N})$, where $\zeta$ is the fine-scale adjustment for a particular echo, $\mathrm{j}=\mathrm{V}_{-}-1$ and $\mathrm{i}$ is an index from 1 to $\mathrm{N}$, with $\mathrm{N}=128$, being the number of frequency samples (waveform bins). Denoting the complex waveform, $\mathrm{C}_{\mathrm{k}}(\mathrm{k}=1,128)$ as the Fourier transform, F[.] of this adjusted signal, and invoking the convolution theorem gives:

$$
\begin{aligned}
\mathrm{C}_{\mathrm{k}} & =\mathrm{F}\left[\left(\mathrm{I}_{\mathrm{i}}+\mathrm{j} \mathrm{Q}_{\mathrm{i}}\right) \cdot \exp (-2 \pi \mathrm{j} \xi \mathrm{i} / \mathrm{N})\right] \\
& =\mathrm{F}\left[\left(\mathrm{I}_{\mathrm{i}}+\mathrm{j} \mathrm{Q}_{\mathrm{i}}\right] * \mathrm{~F}[\exp (-2 \pi \mathrm{j} \xi \mathrm{i} / \mathrm{N})]\right.
\end{aligned}
$$

where ${ }^{\prime *}$ ' implies convolution.

If $\zeta$ is an integer, the Fourier transform of the second part of (2) is just a Dirac $\delta$ function corresponding to a simple translation of the waveform by $\zeta$ bins. If $\zeta$ is not an exact integer, which is generally the case, then the second part of (2) provides a complex interpolation, which approximates to moving the leading edge by $\zeta$ bins. The permitted range for $\zeta$ is up to 6 bins, with the actual value quantized to span this interval in 256 equal steps. If the expected return would require a shift outside this range, then the timing of the secondary chirp used in the deramping is advanced or receded by the equivalent of 6 bins, which is termed the coarse-scale adjustment. The average echo is then the sum of the power over 96 individual waveforms:

$$
\mathrm{AE}_{\mathrm{k}}=\sum_{\mathrm{m}} \mathrm{C}_{\mathrm{m}, \mathrm{k}} \mathrm{C}_{\mathrm{m}, \mathrm{k}}^{\dagger}
$$

where $\mathrm{k}$ denotes waveform bin number (1-128), $\mathrm{m}$ is the numbering of successive waveforms, and ${ }^{\dagger}$ denotes complex conjugate. In normal operations, only these AEs are kept and telemetered to the ground.

However, at specific times (irregularly, but averaging 4 times a day) a 1-second burst of IEs is recorded and transmitted in its entirety. This allows an independent investigation of the on-board processing and also the opportunity to investigate the potential for different processing strategies. Typically this burst will consist of 42 segments of 96 IEs, thus corresponding to $1.09 \mathrm{~s}$ of data $(\sim 7.1 \mathrm{~km}$ distance along track). The data provided in this high density (HD) product are the I and Q component of the original received echo in frequency space, plus the power, corresponding to $C_{k} C^{\dagger}{ }_{k}$ i.e. the $I^{2}+Q^{2}$ term after adjustment by fine scale shift, $\zeta$, and application of Fourier transform. 
We were granted access to a limited number of these HD products. In this paper we concentrate on the 17 unique bursts we had over marine surfaces for which the mean shape is well described by the Brown-Hayne model, and make a preliminary investigation of the extra detail within these individual echoes.

\section{Shape and characteristics of mean waveform}

In this section and the succeeding one we concentrate on the power signal within the individual echoes. The so-called "average echoes" actually correspond to the total waveform power received within a segment, rather then the mean of the power in those 96 individual echoes. First of all we assess the efficacy of the on-board processing by comparing the average echoes to the sum of the power in the IEs in each segment. Figure 2a shows a typical result for one segment; note there are only 104 bins in the telemetered AE, corresponding to bins 13-116 of the IEs. Aside from these omitted bins, no difference is perceptible. In fact, there is a difference in that the $\mathrm{AE}$ values have been rounded to the nearest integer prior to transmission, whereas the sum of the IEs is, in general, a non-integer. This distinction becomes more important later.

Figure $2 \mathrm{~b}$ shows the results for all 42 segments in a burst, with the mean of the AEs and the mean of the summed IEs. (Each segment will have an associated setting, specifying the value of the automatic gain control (AGC) during its collection. For the examples illustrated here, bursts have been chosen for which all 42 AGC settings are the same, so that its implementation is not pertinent to the discussion.) The juxtaposition of the two lines confirms the correctness of the on-board implementation. Plotting the ordinate as $\log _{10}(\mathrm{P})$, where $\mathrm{P}$ is the power in the waveform bin, gives a fresh insight into the shape of AltiKa's mean waveforms (Figure 2c). As expected the trailing edge is very well approximated by a linear fit; however there is also a linear growth within the thermal noise region, which is not expected. The "wrap-round" effect in the first few and last few bins is now quite clear; this is due to the circular Fourier transform used to convert the I \& Q components into the complex waveform combined with the anti-aliasing filter that suppresses the signal beyond the waveform window (Chelton et al., 1989). We categorise this wrap round effect as "pronounced" when the burst mean differs from the linear fit by 0.0107 in $\log _{10}(\mathrm{P})$, equivalent to a factor of $2.5 \%$ in absolute power terms.

Table 1 details the slopes found in these two sectors of the waveform and how many bins deviate from this fit. The slope in the trailing edge region is principally due to the antenna beamwidth, although it might also be affected by instrument mispointing if present, or by irregularities within the on-board filter (Desjonqueres, pers. comm., 2014). The cause of the signal in the thermal noise region is unknown, although the response of the intermediate frequency filter has been suggested (Poisson, pers. comm. 2014). Although both slopes show some variation (see error bars in Table 1), there is no correlation between the two. Finally, it is noted that typically 8 bins are affected by wrap-round at the front of the waveform, and 12 at the rear. With the variation shown by the error bars, the excision of the first 12 and last 12 bins from the standard waveform product (AEs) seems fully justified.

In standard waveform retracking, assumptions are made about the independence of the signal at consecutive waveform bins, and that the pulses contributing to the AE are all independent. Quartly et al (2001) evaluated this latter assumption for many of the early altimeters. They considered continuous stretches of homogenous data (little variation in $\mathrm{H}_{\mathrm{s}}$ or $\sigma^{0}$ ), and calculated the mean and the variability of the AEs. The ratio of the mean to the standard deviation (s.d.) can be shown to be $\sqrt{N}_{0}$, where $\mathrm{N}_{0}$ is the number of independent pulses.

We performed such analysis for the 42 AEs in a burst (noting that the same AGC setting was applicable to all). The result (Fig. 2d) shows a plateau close to $\sqrt{96}$ in the trailing edge of the waveform, but with much lower values in the thermal noise region. This is surprising in that every realization of thermal noise should be completely independent, unless there was some interference or power leakage within the altimeter waveguide. Similar results had been noted for ERS-1, ERS-2, TOPEX and Poseidon (Quartly et al. 2001). However, with AltiKa we find the expected performance can be achieved if the summed IEs are used instead. The distinction is that the data within the AEs has been quantised in counts, and this can be demonstrated by rounding of the summed IEs to the nearest integers before 
independence analysis. At the toe of the leading edge the situation is more complicated to interpret such early returns correspond to reflections from a small disk on the surface, that may decorrelate less rapidly than for a large footprint, but there may also be effects of incomplete correction for waveform positioning due to tracker movement. In normal operations the peak of the summed waveform corresponds to more than 200 counts; in such cases rounding of values to the nearest integer has negligible impact upon retracking of range and $\mathrm{H}_{\mathrm{s}}$. However, the quantization of data may impact recovery of sea surface skewness, which has its most pronounced effect near the toe of the waveform's leading edge.

\section{Finer spatial sampling}

For oceanographic applications $40 \mathrm{~Hz}$ data is sufficient, corresponding to along-track sampling of $\sim 174 \mathrm{~m}$, especially given that the instrument footprint corresponds to a disk with a minimum diameter of $2 \mathrm{~km}$. However over much more variable terrain, such as over lakes, rivers, and in coastal areas where calm water and land returns in the footprint can influence the shape of the returned echo (Gómez-Enri et al. 2010), it may be advantageous to use data at a finer resolution, allowing the removal of signals whose waveform shape does not match expectation. To evaluate this potential we construct our own AEs from the IE powers within a burst over the ocean. In these idealised conditions, away from extraneous reflections from land surfaces, we can investigate how tracker performance is degraded as we move to noisier AEs calculated from fewer IEs.

We perform this evaluation with the adaptive leading-edge subwaveform (ALES) retracker (Passaro et al. 2014). This optimises the fit to a subwaveform i.e. those bins nearest to the leading edge, with the number of bins employed adjusted according to an initial estimate of the wave height. We use a subwaveform retracker firstly because even with only a few IE powers summed the leading edge is welldefined, whereas the trailing edge contains many spurious peaks (see Fig. 3a). Secondly, we wish to evaluate the subwaveform retracker because it is the kind of algorithm that would be used over coastal or inland waters, where using only a selection of IEs might be appropriate. ALES has been shown to work very well in the coastal zone, where it offers resilience to the effect of spurious extra reflections appearing within the waveform trailing edge, whilst over the open ocean it does not degrade the accuracy of the estimation with respect to that of a full waveform fitting. In this paper, the comparison is not with independent ground truth, but rather how consistent the retracking of our new AEs is with the results obtained from the standard $40 \mathrm{~Hz}$ product made from 96 IEs at a time.

As the bandwidth of AltiKa's emitted chirps is greater than that used in $\mathrm{K}_{\mathrm{u}}$-band altimeters, the width of the bins within the waveform is smaller ( $\sim 2.1 \mathrm{~ns}$ as opposed to $3.125 \mathrm{~ns})$ corresponding to a range increment of $30 \mathrm{~cm}$. However, to achieve the sub $10 \mathrm{~cm}$ accuracy usually required from altimeters nowadays still requires pinpointing the leading edge to a fraction of the binwidth. Although a single IE does have a definite leading edge (see Fig. 1a) the expected variability at any particular bin precludes it from giving the desired accuracy. The more IEs that are summed to produce an average, the better defined will be its leading edge and the less noisy its trailing edge (Fig. 3a). The question is how many IEs should be summed to form a waveform that can be satisfactorily retracked by ALES.

To give a quantitative answer, for each $0.025 \mathrm{~s}(40 \mathrm{~Hz})$ segment we can thus construct a number of AEs that depends on how many IEs are summed for each of them. This processing is applied to our 17 burst files over the ocean, with the putative AEs calculated from 8, 12, 16, 24 and 32 IEs. The ALES retracker is applied to the $40 \mathrm{~Hz}$ waveforms and to the different AEs to evaluate the retracking performances. Two statistics are taken into account for each $0.025 \mathrm{~s}$ segment for both the estimation of range and of $\mathrm{H}_{\mathrm{s}}$ :

1) the median absolute bias of the estimations from each $\mathrm{AE}$ with respect to the estimation from the $40 \mathrm{~Hz}$ waveform, and

2) the standard deviation of the AEs estimations within the whole burst. 
The first is a measure of uncertainty related to a "best case" estimation, in this case the retracking performed on the average of all the 96 IEs of each segment. The second is a measure of noise in the estimation.

Results are shown in Figure 3b-e for range and $\mathrm{H}_{\mathrm{s}}$ estimation. Median results from each file are colour-coded depending on the number of IE powers averaged before retracking. The statistics are plotted against the median $\mathrm{H}_{\mathrm{s}}$ of each burst, estimated from ALES applied to the $40 \mathrm{~Hz}$ waveforms. As expected, this demonstrates that more precise results are achieved at lower values of $\mathrm{H}_{\mathrm{s}}$. Precision also improves with increasing number of IEs averaged, but it is surprising to see how consistent the estimations can be even at very high frequencies. For example, the ALES retracking of sums of 32 IEs $(120 \mathrm{~Hz})$ adds a lower noise level to epoch estimation than was estimated for $10 \mathrm{~Hz}$ AEs for Jason-1 (Zanife et al. 2003). Generally, the findings are consistent with the familiar picture from past altimetry missions of greater range uncertainty in $1 \mathrm{~Hz}$ records at larger $\mathrm{H}_{\mathrm{s}}$.

Table 2 provides the median values for bias and precision across these diverse 17 cases. These show that the error in the tracking of a generated average echo typically scales as $\mathrm{M}^{-0.5}$, where $\mathrm{M}$ is the number of IEs summed together. This is as expected from statistical considerations for the mean calculated from $\mathrm{M}$ independent samples. Similarly, the precision, here estimated as the s.d. of the estimates, scales as $\mathrm{M}^{-0.5}$.

An application emerging from this analysis is the possibility to retrack waveforms at a frequency $>$ $40 \mathrm{~Hz}$ and eliminate the AEs that produce an estimate substantially different from the rest within a 1second burst. Corrupted high-rate AEs could therefore be identified as outliers and the remaining estimates can then be averaged to produce a more robust result at a lower frequency. This procedure could be useful in coastal areas and inland water bodies to eliminate the IEs that are highly corrupted by land and coastal waters, but has not been evaluated yet as none of the burst records we received has been in the coastal zone.

\section{Information within complex echoes}

The individual echoes also offer the potential of new types of analysis based on the phase information available and also on the correlation between successive waveforms. We provide here a cursory examination of the phase information within AltiKa, which may serve as a pointer for other investigators. Successive waveforms will be independent if in the time between them the range of the satellite to reflecting facets on the sea surface changes by a significant fraction of the radar wavelength. Walsh (1982) gave an analytical form for this, and predicted that, for a $\mathrm{K}_{\mathrm{u}}$-band altimeter in an orbit similar to Envisat's, pulses at a PRF of $2 \mathrm{kHz}$ would only be definitely independent for wave heights greater than $5 \mathrm{~m}$. (This threshold scales with $\sqrt{H}_{\mathrm{s}}$, and linearly with the radar frequency.)

SAR altimetry processing, which relies on the phase coherence to Doppler filter the return echoes and thus achieve a better range accuracy, operates at much higher values of the PRF e.g. $18200 \mathrm{~Hz}$ for Cryosat-2 (Wingham et al. 2006). Nevertheless various efforts were made with the Envisat altimeter to examine whether extra information was contained within its IEs. The $\mathrm{K}_{\mathrm{u}}$-part of the Envisat dualfrequency altimeter operated at a PRF of $1800 \mathrm{~Hz}$, and routinely collected 1- second bursts of IEs every 3 minutes from 2004 to its demise. The RAIES (Radar Altimeter Individual Echoes and S-band) project explored the use of IEs over a number of surfaces showing useful waveforms could be constructed from as few as 5 pulses (Gommenginger and Quartly 2007). Patterns were found within the phase information at neighbouring bins, but that was ascribed to the influence of a Hamming filter at the beginning of the reception chain i.e. before the IEs were recorded. Gommenginger and Quartly (2007) also calculated the phase structure function, but the hoped for connection to ocean wave parameters was not proven. Coherent phase information was demonstrated by Quartly (2010) in a number of case studies, but the echoes were interpreted as coming from either a ship superstructure or from a strong reflector over land. Such results indicate that the phase information of the IEs has been adequately recorded and retained through the processing chain, but does not advance oceanographic study. Recently Abileah et al. (2013) examined the correlation between successive waveforms by calculating the complex cross product and summing over all waveforms in the burst: 


$$
\mathrm{Z}_{\mathrm{k}}=\sum_{\mathrm{m}} \mathrm{C}_{\mathrm{m}, \mathrm{k}} \mathrm{C}_{\mathrm{m}+1, \mathrm{k}}^{\dagger}
$$

where $\mathrm{k}$ is the index over waveform bins and $\mathrm{m}$ is that over waveforms, and the defined term, $\mathrm{Z}$, is the 1lag covariance. Abileah et al (2013) showed that the phase of this in the primary peak (nadir reflections) was related to the Doppler velocity.

Figure $4 \mathrm{a}$ shows the phase of the lagged complex cross product, $C_{m, k} C_{m+1, k}^{\dagger}$, Some structure is visible both in the vertical bands within the trailing edge (bins 60 to 100), as well as occasional horizontal bars of fixed phase difference corresponding to bins near the toe of the leading edge. These two features are enhanced in the succeeding plots.

First Figures $4 \mathrm{~b}$ and $4 \mathrm{c}$ show the amplitude and phase of $\mathrm{Z}$, with the phase of the summation being bimodal in the trailing edge region, with peak values $\pi$ apart. These variations within the waveform correspond to points in an Argand diagram close to an oblique line through the origin, whose slope is related to the Doppler velocity. The amplitude variations of $\mathrm{Z}$ are governed by the Bessel function, $\mathrm{J}_{0}$, whose argument is proportional to the radius of the annulus of reflecting facets (W. Smith, pers. comm., 2013), and so does not appear regular when plotted against bin number. From an analysis of Cryosat-2 data, Smith and Scharroo (2012) argued that whilst reflections from most of the pulse-limited footprint decorrelate rapidly as foretold by Walsh (1982), there can be a strip of zero-Doppler signal some $300 \mathrm{~m}$ across that will remain phase-coherent for much longer. Thus the apparent contradiction between independence of the power in successive IEs (Fig. 2d) and the complex coherence (Fig. 4b,c) may be explained by the constancy of the phase offset of returns from this section of the altimeter footprint.

To highlight the significance of the horizontal bands of constant phase offset in Figure 4a, we look at the phase difference, $\Delta \Phi$, between successive bins in the same waveform. If there is no relationship between the phases at different bins then $\Delta \Phi$ will have a uniform distribution between $-\pi$ and $\pi$, which has a mean of 0 and a s.d. of $\pi / \sqrt{ } 3$. This is the case for bins within the leading edge and trailing edge of the waveform where the majority of the signal is. However, there is a marked departure around bins 45 to 50 , which is very much the toe of the trailing edge (power only a few $\%$ of that at peak of waveform). This is shown in more graphic detail by the histogram of the phase difference between bins 49 and 50 (Figure 4f). The interpretation of this is unclear at the moment, but we note that such returns would correspond to the extreme peaks of waves within the immediate footprint, and thus represent a relatively small number of scattering surfaces. There is also a weak departure from expected behaviour in the thermal noise region, which is most clear in the mean of $\Delta \Phi$ (Figure $4 \mathrm{~d}$ ).

\section{Summary and conclusions}

In this paper we have made an initial evaluation of the individual echoes from AltiKa. First we were able to demonstrate that the average echoes, which are a standardly available product, are consistent with the records of power for the IEs, except that the AE product involves rounding of the data to integer values and the removal of the first 12 and last 12 bins. A detailed investigation of the shape of the mean waveform revealed an unexplained exponential increase in power in the thermal noise region (a linear increase in $\log (\mathrm{P}))$. The waveform wrap-round effect engendered by the anti-aliasing filter and the circular nature of the Fourier transform was quite clear in the mean profile of the IEs; this agreed with the policy of removing such bins from the standard AE product. Access to the more detailed IEs enabled us to confirm that previous analyses on the independence of pulses had given spurious values in the thermal noise region (Quartly et al. 2001) due to the quantization of records. At the beginning of the leading edge the sampling variability appears to be a greater proportion of the mean than elsewhere within the waveform (inverse of Figure 2d); this finding is relevant for retracking techniques that treat the fitting error as proportional to the expected signal (see section 4.6.2 of Gommenginger et al. 2011).

Secondly, we confirmed the implementation of the fine scale adjustment to the I and Q frequency components in order to match with the power signal in the waveforms. Although the PRF is around the maximum rate that Walsh (1982) asserted could still be treated as independent, our preliminary analysis of the complex echoes did show some structure to be present within the phase information. Calculation of the 1-lag covariance, as advocated by Abileah et al. (2013) showed a summation vector for each 
waveform bin whose amplitude was a series of peaks, with the phase flipping by $\pi$ between each lobe, with the particular phases being dependent upon the rate at which the spacecraft was approaching or receding from the Earth's surface. A strong consistency in the phase offset of succeeding complex IEs was also noted at the very toe of the leading edge, which would correspond to a relatively small reflecting surface. With the limited number of IE bursts that we had, it was not possible to relate these intriguing findings to any physical cause. Possibly an examination of IE bursts over strong discrete reflecting surfaces (as investigated by Quartly (2010) for Envisat) may help explain these results. The investigation of IEs by other research groups would be helped by better documentation and a HD product that included the complex waveforms.

Finally, and perhaps most importantly, we have demonstrated that average echoes constructed from just a few IEs can be tracked with a noise level comparable to what was a mission target at $1 \mathrm{~Hz}$ only a decade ago. For example, at low to moderate wave conditions the noise on range estimation at $120 \mathrm{~Hz}$ can be kept below $5 \mathrm{~cm}$. Our analysis used the ALES retracker (Passaro et al. 2014), which is designed to take most heed of the leading edge and not be affected by spurious returns in the trailing edge. As such it is ideal for application across narrow water bodies, for which even a $40 \mathrm{~Hz}$ product may be too coarse, and along the coast, where retracking of high-frequency waveforms could help identifying and discarding the AEs that are corrupted by spurious reflections in order to create more reliable $40 \mathrm{~Hz}$ estimations.

\section{Acknowledgements}

This work was carried out as part of the KaNUTE project. The authors are grateful to Amandine Guillot \& Nicolas Picot for the provision of the High Density (HD) data, to Jean-Damien Desjonquères for useful discussions at the SARAL/AltiKa Meet, and to Helen Snaith for comments on a draft of this manuscript. Marcello Passaro is also grateful to Jerome Benveniste for his support.

\section{References}

Abileah, R., J. Gómez-Enri, A. Scozzari, and S. Vignudelli, 2013, Coherent ranging with Envisat radar altimeter: A new perspective in analyzing altimeter data using Doppler processing, Remote Sensing of Environment 139: 271-276. doi: 10.1016/j.rse.2013.08.005

Brown, G.S., 1977, The average impulse response of a rough surface and its applications IEEE Transactions on Antennas and Propagation 25: 67-74.

Chelton, D.B., E.J. Walsh, and J.L. MacArthur, 1989. Pulse Compression and Sea Level Tracking in Satellite Altimetry. Journal of Atmospheric and Oceanic Technology, 6: 407-438.

Hayne, G.S., 1980, Radar altimeter mean return waveforms from near-normal-incidence ocean surface scattering, IEEE Transactions on Antennas and Propagation 28: 687-692.

Gómez-Enrí, J., S. Vignudelli, G.D. Quartly, C.P. Gommenginger, P. Cipollini, P.G. Challenor and J. Benveniste, 2010. Modeling Envisat RA-2 waveforms in the coastal zone: Case-study of calm water contamination, IEEE Geoscence and Remote Sensing Letters, 7: 474-478. doi: 10.1109/LGRS.2009.2039193

Gommenginger, C., and G. Quartly. 2007. Third and Final CCN update of NOC RAIES Technical Note on Task 5 "Scientific Exploitation of Individual Echoes for Ocean applications", 55 pp. [ESA contract no. 17900/03/I-LG ; available from European Space Agency]

Gommenginger, C., P. Thibaut, L. Fenoglio-Marc, G. Quartly, X. Deng, J. Gómez-Enri, P. Challenor, Y. Gao, 2011, Retracking altimeter waveforms near the coasts A review of retracking methods and some applications to coastal waveforms, p 61-101 of "Coastal Altimetry" (ed. Vignudelli, S., A.G. Kostianoy, P. Cipollini \& J. Benveniste) Springer, ISBN 978-3-642-12796-3

Passaro, M., P. Cipollini, S. Vignudelli, G.D. Quartly, and H.M. Snaith, 2014, ALES: a multi-mission adaptive sub-waveform retracker for coastal and open ocean altimetry, Remote Sensing of Environment 145: 173-189. doi: 10.1016/j.rse.2014.02.008

Quartly, G.D., 2009, Optimizing $\sigma^{0}$ information from the Jason-2 altimeter. IEEE Geoscence and Remote Sensing Letters, 6: 398-402. doi: 10.1109/LGRS.2009.2013973

Quartly, G.D., 2010, Hyperbolic retracker: removing bright target artefacts from altimetric waveform data. In, ESA Living Planet Symposium, Bergen, 28th Jun-2nd July 2010. Noordwijkerhout, NL, European Space Agency ESA SP-686. (6pp) 
Quartly, G.D., M.A. Srokosz, and A.C. McMillan, 2001, Analyzing altimeter artefacts: statistical properties of ocean waveforms, Journal of Atmospheric and Oceanic Technology 18: 2074-2091.

Smith, W.H.F., and R. Scharroo, 2012. Pulse-to-pulse correlation in CryoSat SAR echoes from ocean surfaces: implications for optimal pseudo-LRM waveform averaging, Presentation at OSTST 2012, Venice, http://www.aviso.altimetry.fr/fileadmin/documents/OSTST/2012/oral/02_friday_28/ 05_instr_processing_IIb/03_IP2B_Smith1.pdf

Walsh, E.J., 1982, Pulse-to-pulse correlation in satellite radar altimeters, Radio Science 17: 786-900.

Wingham, D.J., C.R. Francisb, S. Bakera, C. Bouzinacb, et al., 2006, CryoSat: A mission to determine the fluctuations in Earth's land and marine ice fields, Advance in Space Research, 37: 841-871.

Zanife, O.-Z., P. Vincent, L. Amarouche, J.P. Dumont, P. Thibaut, S. Labroue (2003) Comparison of the Ku-band range noise level and the relative sea-state bias of the Jason-1, TOPEX, and Poseidon-1 radar altimeters, Marine Geodesy. 26: 201-238. doi: 10.1080/01490410390255737

\section{Tables}

Table 1. Characteristics of logarithm of mean waveform. (Error bars are 1 s.d.)

\begin{tabular}{|l|l|}
\hline Slope in thermal noise region & $0.0027 \pm 0.0006$ \\
\hline No. of bins exceeding line & $8.1 \pm 1.2$ \\
\hline Slope in trailing edge region & $-0.0154 \pm 0.0004$ \\
\hline No. of bins below line & $12.4 \pm 0.9$ \\
\hline
\end{tabular}

Table 2. Median error over all 17 bursts as a function of number of IEs summed. The s.d. indicates the consistency of estimates within a burst; the bias is the median absolute deviation with respect to tracking of $40 \mathrm{~Hz}$ waveforms.

\begin{tabular}{|c|c|c|c|c|}
\hline No. of IEs & $\begin{array}{c}\text { Range s.d., } \\
\sigma_{\mathrm{h}}(\mathrm{cm})\end{array}$ & $\begin{array}{c}\text { Range bias, } \\
\Delta \mathrm{h}(\mathrm{cm})\end{array}$ & $\begin{array}{c}\mathrm{H}_{\mathrm{s}} \text { s.d., } \\
\sigma_{\mathrm{Hs}}(\mathrm{m})\end{array}$ & $\begin{array}{c}\mathrm{H}_{\mathrm{s}} \text { bias, } \\
\Delta \mathrm{H}_{\mathrm{s}}(\mathrm{m})\end{array}$ \\
\hline 8 & 16.2 & 8.55 & 0.95 & 0.46 \\
\hline 12 & 14.4 & 7.90 & 0.71 & 0.40 \\
\hline 16 & 11.8 & 6.75 & 0.57 & 0.36 \\
\hline 24 & 10.8 & 5.81 & 0.48 & 0.28 \\
\hline 32 & 8.7 & 4.34 & 0.45 & 0.24 \\
\hline
\end{tabular}




\section{Figures}
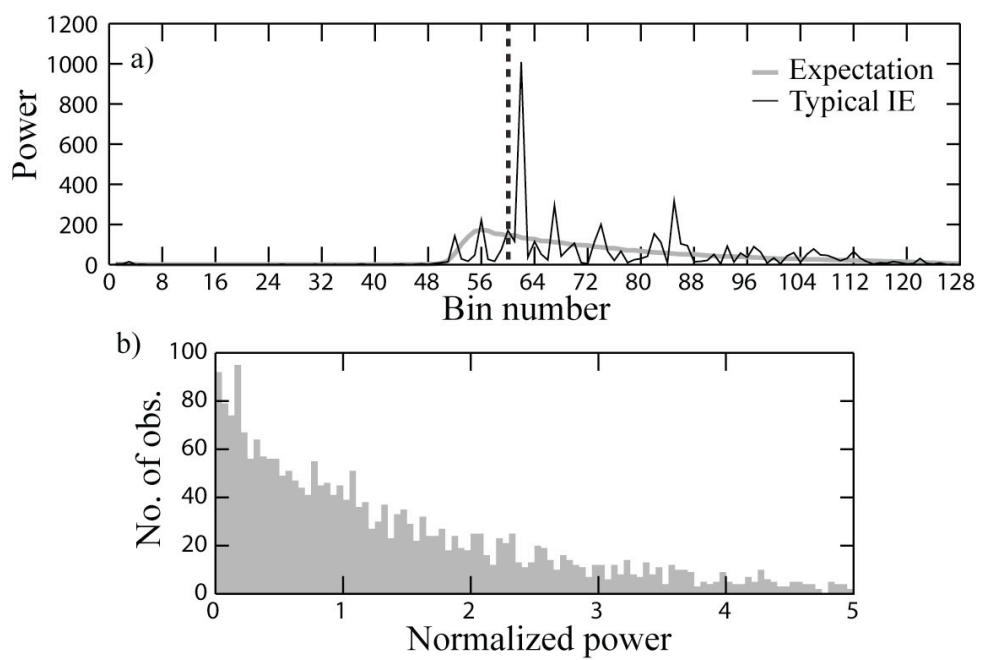

Figure 1. a) Shape of mean (expected) waveform, compared with the echo of a single pulse. (The IE has been multiplied by 96 to match the scaling of the expectation (mean AE in the burst). b) Histogram of power observed at bin 60 from one burst (4032 samples), normalized by the mean value at that bin.

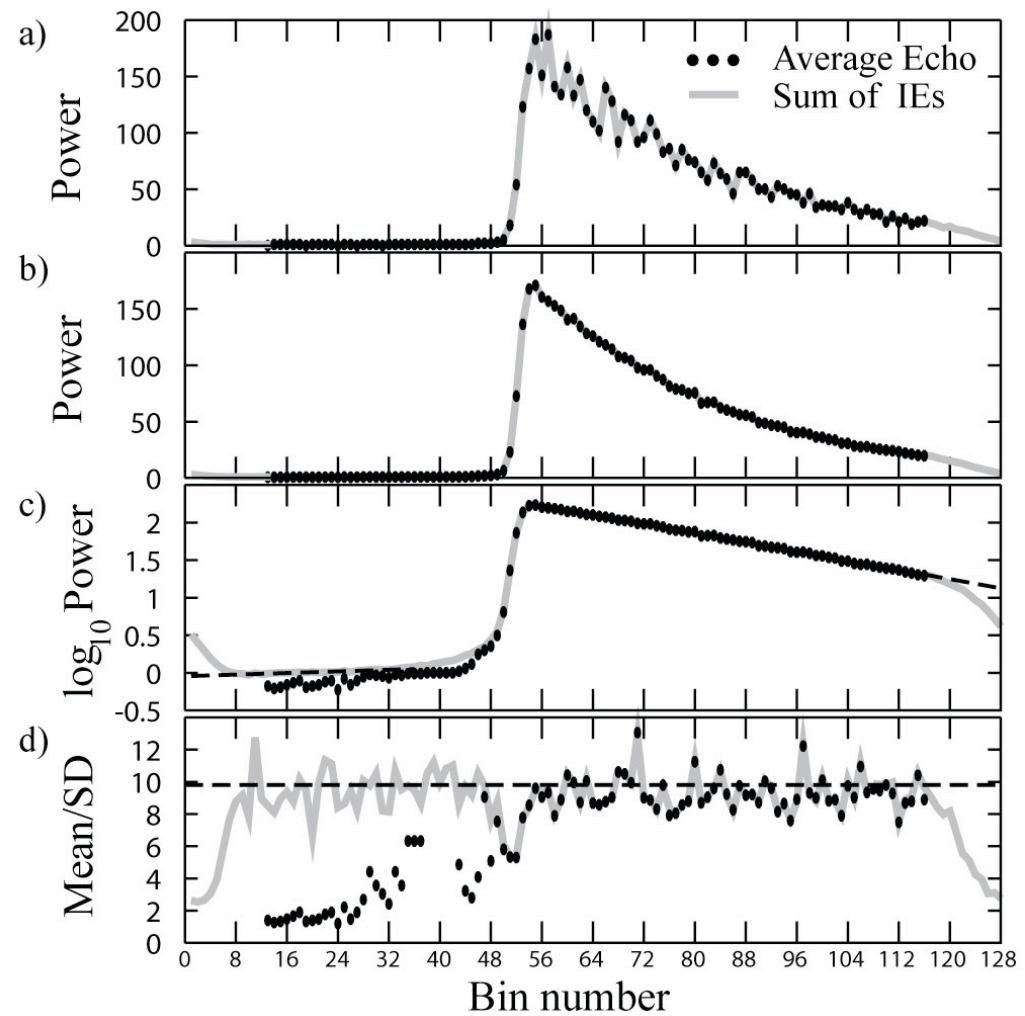

Figure 2. Comparison of sum of 96 IEs with the relevant AE. a) Results for one segment. b) Mean of 42 segments in a burst. c) As in b) but with logarithmic scale and lines fitted to IEs for bins 12-30 and 60110. respectively. d) Using mean waveforms for each segment the ratio of the mean to s.d. is plotted for each bin (dashed line is $\sqrt{ } 96)$. 

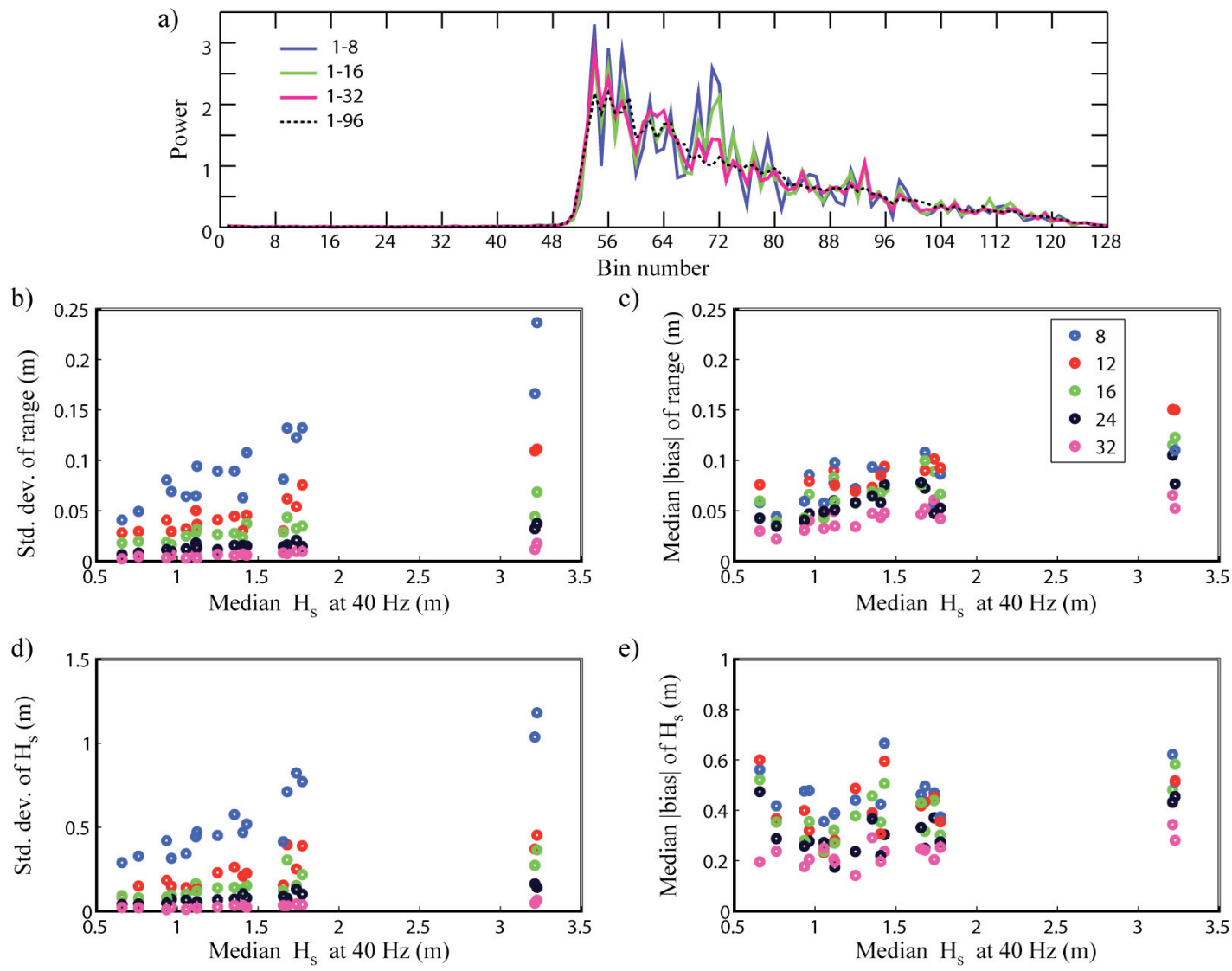

Figure 3. Tracking error engendered by summing fewer IEs. a) Illustration that the leadined edge is welldefined by just a few summed pulses, whereas the trailing edge is much less clear. b), d) Consistency of retracking of range and $\mathrm{H}_{\mathrm{s}}$ respectively within a 1s burst. c), e) Mean absolute bias (relative to processing of $40 \mathrm{~Hz}$ waveforms) for range and $\mathrm{H}_{\mathrm{s}}$. 

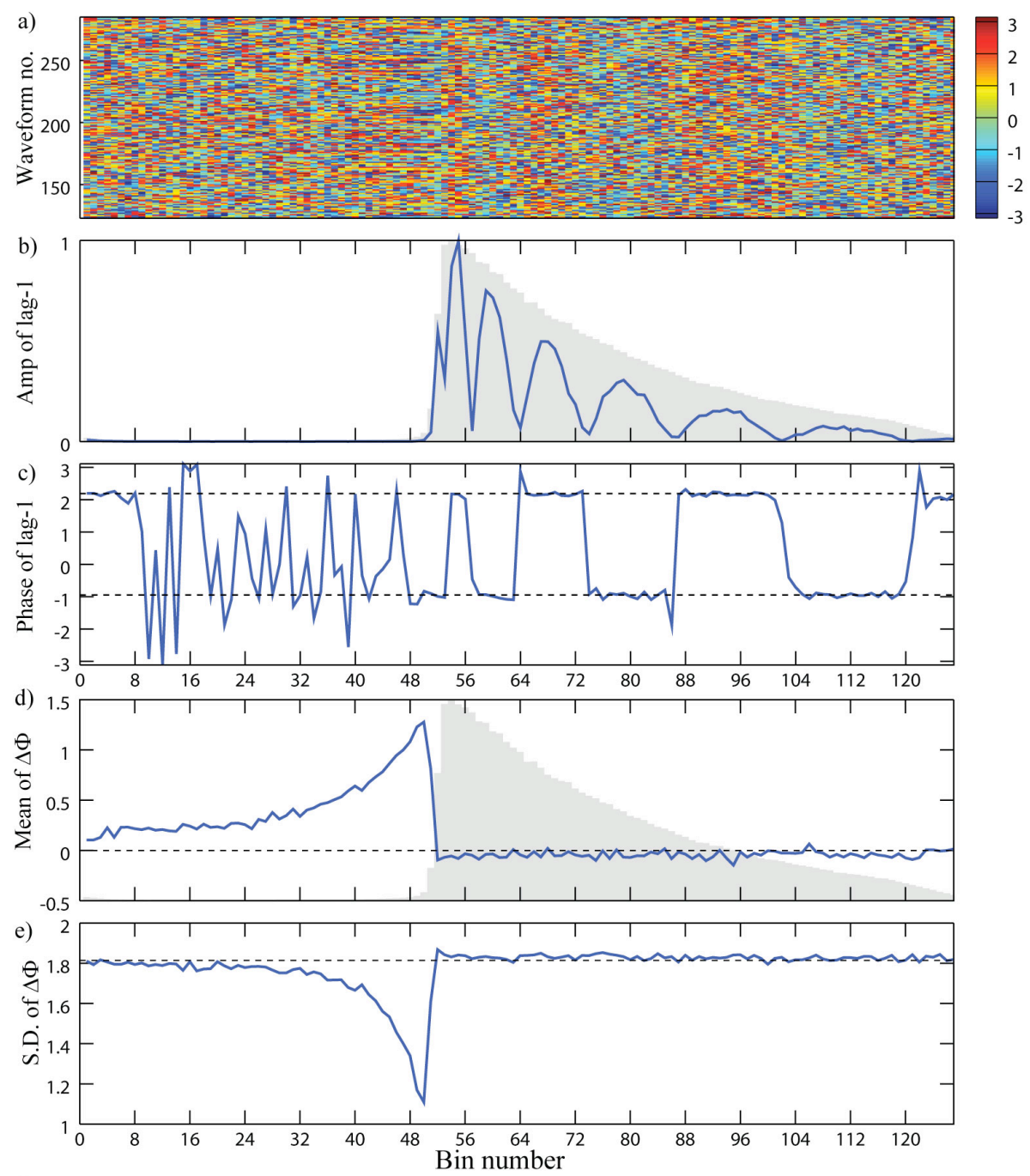

f)

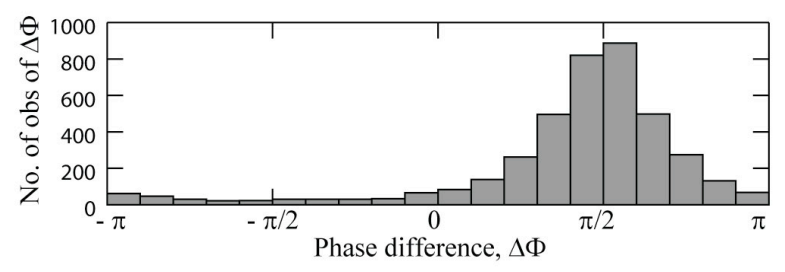

Figure 4. Illustration of phase relationships within a 1-seceond burst. a) Section of phase change between successive complex IEs. b), c) Amplitude and phase of lag-1 covariance, Z. d), e) Mean and variability (s.d.) of phase change, $\Delta \Phi$, between successive bins in same waveform. f) Histogram of phase change between 49th and 50th bins of selected burst. The light grey shading in b), d) indicate the position of the mean waveform power, and the dashed line in e) is at $\pi / \sqrt{ } 3$. 